\title{
Clinical Evaluation of Factors To Predict Successful Vaginal Birth After Cesarean (Vbac) in Cases of Previous one Lower Segment Cesarean Section (Lscs)
}

\author{
Dr. Sarita Sinha ${ }^{1}$, Dr. Devender Kumar ${ }^{2}$, Dr. Anjali Tempe ${ }^{3}$, \\ Dr. Harendra Kumar ${ }^{4}$ \\ ${ }^{1}$ (Obstetrics And Gynaecology,Resident,Mamc/Delhi,India) \\ 2 ( Obstetrics And Gynaecology, Professor,Mamc/Delhi, India ) \\ ${ }^{3}$ ( Obstetrics And Gynaecology,Hod,Mamc/Delhi, India ) \\ ${ }^{4}$ (Orthopaedics, Resident, Bmch/W.B., India )
}

\begin{abstract}
The increasing trend of LSCS is a public as well as health professionals concern, out of which repeat LSCS accounts for a major chunk. Our study was designed to evaluate the clinical factors as predictors in successful cases of VBAC after one LSCS done for non-recurrent indication.

A prospective observational study comprising of 500 patients was performed. 50\% of TOLAC cases had successful vaginal delivery. $86 \%$ of successful VBAC was observed when period of gestation was 40 weeks or less. $82 \%$ of cases who had previous VBAC had successful TOLAC. 54\% of patients who delivered vaginally were third or higher gravidae. Patients having spontaneous onset of labour had better chances of vaginal delivery. Duration of augmentation with oxytocin also played an important role in predicting successful VBAC. Out of the 250 cases who delivered vaginally $84.4 \%$ had babies weighing $2-3.5 \mathrm{~kg}$. Our study concluded that VBAC success can be predicted by certain clinical factors like prior VBAC, period of gestation, estimated fetal weight, spontaneous onset of labour, gravidity, regarding which the couple should be counselled. TOLAC is safe and can reduce repeat cesarean section and thus reduce maternal morbidity and mortality.
\end{abstract}

Keywords: Cesarean section, factors, TOLAC, VBAC, vaginal delivery.

\section{Introduction}

VBAC is cost - effective and has reduced risk of haemorrhage, postpartum fever, wound sepsis, endometritis, blood transfusion and fewer neonatal problems compared to repeat LSCS.[1]If there are no contraindications, a woman with previous LSCS should be offered trial of vaginal delivery. This will reduce the chances of repeat LSCS and thus complications like adherent placenta. Still clinicians are offering repeat LSCS due to fear which is not well supported with clinical evidence. In fact there are no randomized controlled trials comparing VBAC with repeat LSCS.[2]Hence, there is a need to understand variables in previous and present pregnancy to predict the success of TOLAC.

\section{Aim}

Aim of the study was to evaluate the clinical factors as predictors in successful cases of VBAC after one previous LSCS.

\section{Materials and methods}

A prospective observational study was undertaken from January 2014 to December 2014 in the department of Obstetrics and Gynaecology at Lok Nayak Hospital (LNH), New Delhi. Total 500 cases of previous one LSCS were recruited for TOLAC. Exclusion criteria were recurrent indication of LSCS, inter pregnancy interval $\leq 12$ months at time of recruitment and other high risk factors (as indication for LSCS).

A detailed obstetric history was taken of all previous pregnancies with special reference to indication of previous cesarean section, post-operative complications, history of blood transfusion, birth weight of baby, inter pregnancy interval, any history of vaginal birth before and after cesarean section. A detailed general examination of woman was done. Routine investigations hemogram, glucose challenge test, HIV, Hbs Ag, urine routine and microscopy examination was noted. Antenatal ultrasound was done. Pelvic assessment was done at term gestation or in labour. An informed consent was taken from all those patients who were counselled for TOLAC. Patients coming to gynae casualty for the first time in labour were examined and mode of delivery was planned accordingly.Patients were monitored in labour room by intermittent auscultation of FHR or by cardiotocogram and vitals charting was done for presence or absence of signs of scar dehiscence.

Details of mode of delivery and neonatal outcome were noted. Patients were also observed for 
postpartum complications. Qualitative variables were compared using Chi- square test/ Fisher's test. P value of $<0.05$ was considered statistically significant.

\section{Results}

50\% ofTOLAC caseshad successful vaginal delivery.

Table 1: Distribution ofageamongtwogroups $(n=500)$

$\mathrm{X} 2=1.025$

\begin{tabular}{|c|c|c|c|c|c|}
\hline \multirow{6}{*}{$\begin{array}{l}\text { Age } \\
\text { groups }\end{array}$} & & \multicolumn{2}{|l|}{ Outcome } & \multirow{2}{*}{ Total } & Pvalue \\
\hline & & Successful VBAC & Failed VBAC & & \multirow{6}{*}{$\mathrm{s} 0.795$} \\
\hline & $<20$ & $1(0.4 \%)$ & $0(0.0 \%)$ & $1(0.2 \%)$ & \\
\hline & $20-30$ & $212(84.8 \%)$ & $214(85.6 \%)$ & $426(85.2 \%)$ & \\
\hline & $31-35$ & $32(12.8 \%)$ & $31(12.4 \%)$ & $63(12.6 \%)$ & \\
\hline & $36-40$ & $5(2.0 \%)$ & $5(2.0 \%)$ & $10(2.0 \%)$ & \\
\hline Total & & $250(100.00 \%)$ & $250(100.00 \%)$ & $500(100.0 \%)$ & \\
\hline
\end{tabular}

$\mathrm{df}=3$

Table 2: Distribution ofsocio-economicstatusamongtwogroups $(\mathrm{n}=500)$

\begin{tabular}{|l|c|c|c|c|c|}
\hline \multicolumn{2}{|c|}{} & \multicolumn{2}{|c|}{ Outcome } & \multirow{2}{*}{ Total } & \multirow{2}{*}{ Pvalue } \\
\cline { 3 - 4 } \multicolumn{2}{|c|}{} & Successful VBAC & Failed VBAC & & \\
\hline \multirow{2}{*}{$\begin{array}{l}\text { Socio } \\
\text { economic } \\
\text { class }\end{array}$} & low & $115(46.00 \%)$ & $87(34.80 \%)$ & $202(40.40 \%)$ & \\
\cline { 2 - 4 } & middle & $130(52.00 \%)$ & $159(63.60 \%)$ & $289(57.80 \%)$ & \multirow{2}{*}{0.032} \\
\cline { 2 - 5 } & upper & $5(2.00 \%)$ & $4(1.60 \%)$ & $9(1.80 \%)$ & \\
\hline Total & $250(100.00 \%)$ & $250(100.00 \%)$ & $500(100.00 \%)$ & \\
\hline
\end{tabular}

$\mathrm{X} 2=6.902 ; \mathrm{df}=2$

Table 3: Distribution ofgravidityamongtwogroups $(\mathrm{n}=500)$

\begin{tabular}{|c|c|c|c|c|c|}
\hline & & \multicolumn{2}{|l|}{ Outcome } & \multirow[b]{2}{*}{ Total } & \multirow[t]{2}{*}{ P value } \\
\hline & & $\begin{array}{l}\text { Successful } \\
\text { VBAC }\end{array}$ & Failed VBAC & & \\
\hline \multirow[t]{6}{*}{ Birth order } & SecondGravida & $115(46.00 \%)$ & $151(60.40 \%)$ & $266(53.20 \%)$ & \multirow{6}{*}{0.038} \\
\hline & ThirdGravida & $90(36.00 \%)$ & $66(26.40 \%)$ & $156(31.20 \%)$ & \\
\hline & FourthGravida & $28(11.20 \%)$ & $23(9.20 \%)$ & $51(10.20 \%)$ & \\
\hline & FifthGravida & $13(5.20 \%)$ & $9(3.60 \%)$ & $22(4.40 \%)$ & \\
\hline & SixthGravida & $3(1.20 \%)$ & $1(0.40 \%)$ & $4(0.80 \%)$ & \\
\hline & SeventhGravida & $1(0.40 \%)$ & $0(0.00 \%)$ & $1(0.20 \%)$ & \\
\hline \multicolumn{2}{|c|}{ Total } & $250((100 \%)$ & $250(100.00 \%)$ & $500(100.00 \%)$ & \\
\hline
\end{tabular}

$\mathrm{X} 2=11.782 ; \mathrm{df}=5$

Table 4: Distribution ofgestational age amongtwo groups

\begin{tabular}{|c|c|c|c|c|c|}
\hline & & \multicolumn{2}{|l|}{ Outcome } & \multirow[t]{2}{*}{ Total } & \multirow[t]{2}{*}{ Pvalue } \\
\hline & & Successful VBAC & Failed VBAC & & \\
\hline \multirow{4}{*}{$\begin{array}{l}\text { Gestation } \\
\text { (weeks) }\end{array}$} & $34-36+6$ wks & $34(13.6 \%)$ & $20(8 \%)$ & $54(10.8 \%)$ & \multirow[t]{5}{*}{0.021} \\
\hline & $37-40$ wks & $181(72.4 \%)$ & $175(70 \%)$ & $356(71.2 \%)$ & \\
\hline & $40+1-41 \mathrm{wks}$ & $32(12.8 \%)$ & $45(18 \%)$ & $77(15.4 \%)$ & \\
\hline & $>41$ weeks & $3(1.2 \%)$ & $10(4 \%)$ & $13(2.60 \%)$ & \\
\hline \multicolumn{2}{|l|}{ Total } & $250(100 \%)$ & $250(100 \%)$ & $500(100 \%)$ & \\
\hline
\end{tabular}

$\mathrm{X} 2=60.801 ; \mathrm{df}=2$ 
Table 5: Distribution ofprevious VBAC

\begin{tabular}{|c|c|c|c|c|c|}
\hline \multicolumn{2}{|c|}{} & \multicolumn{2}{|c|}{ Outcome } & \multirow{2}{*}{ Total } & \multirow{2}{*}{ P value } \\
\cline { 2 - 5 } \multicolumn{2}{|c|}{} & Successful VBAC & Failed VBAC & \\
\hline \multirow{3}{*}{$\begin{array}{c}\text { Previous } \\
\text { VBAC }\end{array}$} & 0 & $190(76.00 \%)$ & $237(94.80 \%)$ & $427(85.40 \%)$ & \\
\cline { 2 - 5 } & 1 & $51(20.40 \%)$ & $12(4.80 \%)$ & $63(12.60 \%)$ & \\
\cline { 2 - 5 } & 2 & $8(3.20 \%)$ & $1(0.40 \%)$ & $1(0.80 \%)$ & \\
\cline { 2 - 5 } & 3 & $1(0.40 \%)$ & $0(0.00 \%)$ & $500(100.00 \%)$ & \\
\hline \multicolumn{2}{|c|}{ Total } & $250(100.00 \%)$ & $250(100.00 \%)$ & \\
\end{tabular}

$\mathrm{X} 2=35.761 ; \mathrm{df}=3$

Table 6: Onset of labour in present pregnancy

\begin{tabular}{|c|c|c|c|c|}
\hline & \multicolumn{2}{|c|}{ Outcome } & \multirow[b]{2}{*}{ Total } & \multirow{2}{*}{$\mathrm{P}$ value } \\
\hline & Successful VBAC & Failed VBAC & & \\
\hline $\begin{array}{l}\text { Cases of Spontaneous } \\
\text { labour }\end{array}$ & $35(14.00 \%)$ & $58(23.20 \%)$ & $93(18.60 \%)$ & 0.008 \\
\hline Induced labour & $215(86.00 \%)$ & $192(76.80 \%)$ & $407(81.40 \%)$ & \\
\hline Total & $250(100.00 \%)$ & $250(100.00 \%)$ & $500(100.00 \%)$ & \\
\hline
\end{tabular}

$\mathrm{X} 2=6.988 ; \mathrm{df}=1$

Table 7:Neonatal outcome (present pregnancy)

\begin{tabular}{|l|l|l|l|l|l|}
\hline \multirow{2}{*}{\multicolumn{2}{|c|}{}} & \multicolumn{2}{|c|}{ Outcome } & \multirow{2}{*}{ Total } & \multirow{2}{*}{ Pvalue } \\
\cline { 2 - 5 } & SuccessfulVBAC & Failed VBAC & & \\
\hline $\begin{array}{l}\text { Neonatal } \\
\text { birth wt. }\end{array}$ & $<2 \mathrm{~kg}$ & $21(8.40 \%)$ & $8(3.20 \%)$ & $29(5.80 \%)$ & \multirow{2}{*}{0.040} \\
\cline { 2 - 5 } & $2-3.5 \mathrm{~kg}$ & $211(84.40 \%)$ & $220(88.00 \%)$ & $431(86.20 \%)$ & \\
\cline { 2 - 5 } & $>3.5 \mathrm{~kg}$ & $18(7.20 \%)$ & $22(8.80 \%)$ & $40(8.00 \%)$ & \\
\hline Total & $250(100.00 \%)$ & $250(100.00 \%)$ & $500(100.00 \%)$ & \\
\hline
\end{tabular}

$\mathrm{X} 2=6.416 \mathrm{df}=2$

\section{Discussion \& Conclusion}

The mean age of patients in our study was 26.4 years in successful VBAC group while 26.8 years in failed VBAC group. We didn't find any statistical significance of maternal age with outcome, however Srinivas et al found that women aged 15-20 years of age were $27 \%$ less likely to have a failed VBAC and women >35 years of age were more likely to experience an unsuccessful trial of labour.[3]

In our study successful VBAC was found in patients those who had weight $<60 \mathrm{~kg}$. Since many of the patients came to casualty in labour as unbooked so, BMI couldn't be calculated. Carroll et al evaluated the VBAC success rate by maternal pre-pregnancy weight.[4] The success rate was $81.8 \%$ for women weighing less than $200 \mathrm{lb}, 57.1 \%$ for women between 200- $300 \mathrm{lb}$ and lowest for women > $300 \mathrm{lb}$ at $13.3 \%$. Durnwald and colleagues also found similar results. [5] Juhasz et al showed the evidence that success rate was $83.1 \%$ and 68.2\% when BMI was less than 19.8 and > 29 respectively.[6] As the BMI increased success rate of VBAC decreased.The low socio- economic group had more successful VBAC while middle class had more failed VBAC. This may be due to higher number of pregnancies and low birth weight seen in that group.

In our study we found that third, fourth, fifth gravida had maximum chances of successful VBAC, while second gravida had less chances of VBAC. Kraiem et al studied 352 women undergoing TOLAC and found that $\geq 3$ increased significantly the probability of success of VBAC. [7]

Women having period of gestation < 40 weeks was found to have higher success rate of VBAC. Quinones et al evaluated 20,156 women of prior cesarean section and the success rate of VBAC for term and pre term was $74 \%$ and $82 \%$ respectively $(\mathrm{p}<0.001)$.[8] Hammoud et al have reported increased rate of scar rupture beyond 40 weeks.[9]

The number of successful VBAC was higher among those who had prior vaginal delivery. The VBAC success rate increased with increasing number of prior VBAC $(63.3 \%, 87.6 \%, 90.9 \%, 90.6 \%$ and $91.6 \%$ for 0 , $1,2,3$ and 4 respectively).Patients those who had spontaneous onset of labor and were admitted to labor room in active stage, had more chances of VBAC.We found that inter-delivery interval had no significant impact on 
successful outcome. Huang et al found VBAC success rate of $79 \%$ for patient having inter pregnancy interval of less than nineteen months, while success rate was $85.5 \%$ for inter delivery interval more than nineteen months.[10]

The successful VBAC was noticed when birth weight was $<3.5 \mathrm{~kg}$ ( $\mathrm{p}$ value $=0.040)$. This was comparable to other studies where birth weight of babies was significantly related to mode of delivery.

Several nomograms or scoring systems were created to predict the VBAC success; but most had limited success.[11] Grobman et al published a nomogram to predict VBAC using six variables mostly related to previous pregnancy, which was found to be very effective in western population.[12]Flamm et al designed a nomogram with present pregnancy variables, which also had good prediction value.[13]There is no such score for South Asian population and USA model was not tested for our population.In our study we observed along with previous as well as present pregnancy factors, other variables like socio-economic status, gestational age at the time of labour, estimated fetal weight and gravidity were also statistically significant variables which were not included in those European nomograms. Taking the western model and our factors we can develop a prediction model for our population. Couple must be counselled regarding these factors and assurance should be given about availability of emergency cesarean services to relieve anxiety. TOLAC is safe and can reduce the need of repeat cesarean section thus reducing maternal morbidity and mortality.

\section{References}

[1]. Landon MB, HauthJC,LevenoKJ,etal.Maternalandperinataloutcomesassociated with a trial of labor after prior cesarean delivery N Engl J Med.2004 Dec16;351(25):2581-9.

[2]. DoddJM,CrowtherCA.Electiverepeatcaesareansectionversusinductionoflabourfor womenwithapreviouscaesareanbirth.CochraneDatabaseSystRev. 2006Oct18(4): CD004906.

[3]. Srinivas SK, Stamilio DM, Sammel MD, Stevens EJ,Peipert JF, Odibo AO, Macones GA.Vaginalbirth aftercaesareandelivery:doesmaternalageaffectsafety andsuccess?PaediatrPerinat Epidemiol.2007Mar;21(2):114-20.

[4]. CarrollCSSr,MagannEF,ChauhanSP,KlauserCK,MorrisonJC.Vaginalbirthafter cesareansectionversuselective repeatcesareandelivery:Weight-basedoutcomes.AmJ Obstet Gynecol.2003Jun; 188(6):1516-20; discussion 1520-2.

[5]. DurnwaldCP, EhrenbergHM, MercerBM.Theimpactofmaternalobesityandweight gain on vaginal birth after cesarean section success. Am JObstetGynecol.2004Sep;191(3):954-7.

[6]. Juhasz G, Gyamfi C, Gyamfi P, Tocce K, Stone JL. Effect of body mass index and excessive weight gain on success of vaginal birth after cesarean delivery. Obstet Gynecol.2005Oct;106(4):741-6.

[7]. Kraiem J, Ben Brahim Y, Chaabane K, Sarraj N, Chiha N, Falfoul A.[Indicators for successful vaginal delivery after cesarean section: a proposal of a predictive score]. Tunis Med. 2006 Jan;84(1):16-20.

[8]. QuiñonesJN, StamilioDM, ParéE, PeipertJF, StevensE, MaconesGA.The effect of prematurity onvaginal birthaftercesareandelivery:successandmaternalmorbidity. Obstet Gynecol.2005Mar;105(3):519-24.

[9]. HammoudA, HendlerI,GauthierRJ, BermanS, SansregretA, BujoldE.Theeffectof gestational age on trial of labor after Cesareansection. JMatern Fetal NeonatalMed.2004 Mar;15(3):202-6.

[10]. HuangWH, NakashimaDK, RumneyPJ,KeeganKAJr, ChanK.Interdeliveryinterval andthesuccessofvaginal birthaftercesareandelivery.ObstetGynecol.2002Jan;99(1):41-4.

[11]. MaconesGA,HausmanN,EdelsteinR, StamilioDM, MarderSJ.Predictingoutcomesof trialsoflabor inwomenattempting vaginalbirthaftercesarean delivery:acomparisonof multivariatemethodswithneuralnetworks.AmJObstetGynecol.2001Feb;184(3):409-13.

[12]. GrobmanWA,LaiY,LandonMB,SpongCYetal.Developmentofanomogramfor predictionofvaginalbirthaftercesareandelivery.ObstetGynecol.2007Apr;109(4):806-12.

[13]. Flamm BL, Geiger AM. Vaginal birth after cesarean delivery: an admission scoring system. Obstet Gynecol. Dec;90(6):907-10. 